\title{
A Note on Hermite-Hadamard Inequalities for Products of Convex Functions
}

\author{
Feixiang Chen \\ School of Mathematics and Statistics, Chongqing Three Gorges University, Wanzhou, Chongqing 404000, China \\ Correspondence should be addressed to Feixiang Chen; cfx2002@126.com \\ Received 14 September 2013; Accepted 17 November 2013 \\ Academic Editor: Maurizio Porfiri \\ Copyright (C) 2013 Feixiang Chen. This is an open access article distributed under the Creative Commons Attribution License, which \\ permits unrestricted use, distribution, and reproduction in any medium, provided the original work is properly cited.
}

We obtain some new Hermite-Hadamard type inequalities for products of convex functions. We conclude that the results obtained in this work are the refinements of the present results.

\section{Introduction}

If $f: I \rightarrow R$ is a convex function on the interval $I$, then, for any $a, b \in I$ with $a \neq b$, we have the following double inequality:

$$
f\left(\frac{a+b}{2}\right) \leq \frac{1}{b-a} \int_{a}^{b} f(t) d t \leq \frac{f(a)+f(b)}{2} .
$$

This remarkable result is well known in the literature as the Hermite-Hadamard inequality.

Since then, some refinements of the Hermite-Hadamard inequality on convex functions have been extensively investigated by a number of authors (e.g., [1-7]).

In [8], Pachpatte established two new Hermite-Hadamard type inequalities for products of convex functions as follows.

Theorem 1. Let $f$ and $g$ be real-valued, nonnegative, and convex functions on $[a, b]$. Then,

$$
\begin{aligned}
\frac{1}{b-a} \int_{a}^{b} f(x) g(x) d x \leq & \frac{1}{3} M(a, b)+\frac{1}{6} N(a, b), \\
2 f\left(\frac{a+b}{2}\right) g\left(\frac{a+b}{2}\right) \leq & \frac{1}{b-a} \int_{a}^{b} f(x) g(x) d x \\
& +\frac{1}{6} M(a, b)+\frac{1}{3} N(a, b),
\end{aligned}
$$

where $M(a, b)=f(a) g(a)+f(b) g(b)$ and $N(a, b)=$ $f(a) g(b)+f(b) g(a)$.
Some new integral inequalities involving two nonnegative and integrable functions that are related to the HermiteHadamard type are also established by many authors. In [9], Pachpatte established some Hermite-Hadamard type inequalities involving two log-convex functions. An analogous result for $s$-convex functions is obtained by Kirmaci et al. in [10]. In [11], Sarikaya presented some integral inequalities for two $h$-convex functions. Some Hermite-Hadamard type inequalities for two operator convex functions are given by Bacak and Türkmen in [12]. For recent results and generalizations concerning Hermite-Hadamard type inequality for product of two functions, see [13] and the references given therein.

In this paper, we obtain new generalizations of HermiteHadamard type inequalities for products of convex functions. This result refines the Hermite-Hadamard type inequalities given in Theorem 1 .

\section{Lemma}

Lemma 2. Let $f$ and $g$ be real-valued, nonnegative, and convex functions on $[a, b]$. If $[c, d] \subset[a, b]$, then

$$
\begin{aligned}
\frac{1}{d-c} \int_{c}^{d} f(x) g(x) d x \leq & \frac{1}{3} M(c, d)+\frac{1}{6} N(c, d), \\
2 f\left(\frac{c+d}{2}\right) g\left(\frac{c+d}{2}\right) \leq & \frac{1}{d-c} \int_{c}^{d} f(x) g(x) d x \\
& +\frac{1}{6} M(c, d)+\frac{1}{3} N(c, d),
\end{aligned}
$$


where $M(c, d)=f(c) g(c)+f(d) g(d)$ and $N(c, d)=$ $f(c) g(d)+f(d) g(c)$.

Proof. We get the conclusions by $f$ and $g$ are nonnegative and convex functions on $[c, d]$ and Theorem 1 .

\section{Main Results}

Theorem 3. Let $f$ and $g$ be real-valued, nonnegative, and convex functions on $[a, b]$. Then,

$$
\frac{1}{b-a} \int_{a}^{b} f(x) g(x) d x \leq L(t) \leq \frac{1}{3} M(a, b)+\frac{1}{6} N(a, b),
$$

where $M(a, b)=f(a) g(a)+f(b) g(b), N(a, b)=f(a) g(b)+$ $f(b) g(a), t \in[0,1]$, and

$$
\begin{aligned}
L(t)= & \frac{t}{3} M(a,(1-t) a+t b)+\frac{t}{6} N(a,(1-t) a+t b) \\
& +\frac{1-t}{3} M((1-t) a+t b, b) \\
& +\frac{1-t}{6} N((1-t) a+t b, b) .
\end{aligned}
$$

Proof. Since $f$ and $g$ are convex on $[a, b]$ and nonnegative, by (2), we get

$$
\frac{1}{b-a} \int_{a}^{b} f(x) g(x) d x \leq \frac{1}{3} M(a, b)+\frac{1}{6} N(a, b) .
$$

Firstly, $f$ and $g$ are convex on $[a,(1-t) a+t b]$ and nonnegative; applying (2) on $[a,(1-t) a+t b]$, we get

$$
\begin{gathered}
\frac{1}{t(b-a)} \int_{a}^{(1-t) a+t b} f(x) g(x) d x \\
\leq \frac{1}{3} M(a,(1-t) a+t b) \\
\quad+\frac{1}{6} N(a,(1-t) a+t b) .
\end{gathered}
$$

Similarly, we can show that

$$
\begin{gathered}
\frac{1}{(1-t)(b-a)} \int_{(1-t) a+t b}^{b} f(x) g(x) d x \\
\leq \frac{1}{3} M((1-t) a+t b, b) \\
\quad+\frac{1}{6} N((1-t) a+t b, b) .
\end{gathered}
$$

Multiplying (8) by $t$ and (9) by $(1-t)$ and adding the resulting inequalities, we get

$$
\begin{aligned}
& \frac{1}{b-a} \int_{a}^{b} f(x) g(x) d x \\
& \leq \frac{t}{3} M(a,(1-t) a+t b) \\
&+\frac{t}{6} N(a,(1-t) a+t b) \\
&+\frac{1-t}{3} M((1-t) a+t b, b) \\
&+\frac{1-t}{6} N((1-t) a+t b, b)=L(t) .
\end{aligned}
$$

Using the fact that

$$
\begin{aligned}
& \frac{t}{3} M(a,(1-t) a+t b)+\frac{1-t}{3} M((1-t) a+t b, b) \\
& =\frac{t}{3}[f(a) g(a)+f((1-t) a+t b) \\
& \times g((1-t) a+t b)] \\
& +\frac{1-t}{3}[f((1-t) a+t b) g((1-t) a+t b) \\
& +f(b) g(b)] \\
& =\frac{t}{3} f(a) g(a)+\frac{1-t}{3} f(b) g(b) \\
& +\frac{1}{3} f((1-t) a+t b) g((1-t) a+t b) \\
& \leq \frac{t}{3} f(a) g(a)+\frac{1-t}{3} f(b) g(b) \\
& +\frac{1}{3}[(1-t) f(a)+t f(b)] \\
& \times[(1-t) g(a)+\operatorname{tg}(b)] \\
& =\frac{t+(1-t)^{2}}{3} f(a) g(a)+\frac{1-t+t^{2}}{3} f(b) g(b) \\
& +\frac{(1-t) t}{3} N(a, b) \\
& =\frac{1-t+t^{2}}{3} M(a, b)+\frac{(1-t) t}{3} N(a, b), \\
& \frac{t}{6} N(a,(1-t) a+t b)+\frac{1-t}{6} N((1-t) a+t b, b) \\
& =\frac{t}{6}[f(a) g((1-t) a+t b)+f((1-t) a+t b) g(a)] \\
& +\frac{1-t}{6}[f((1-t) a+t b) g(b) \\
& +f(b) g((1-t) a+t b)]
\end{aligned}
$$




$$
\begin{aligned}
& \leq \frac{t}{6}[f(a)((1-t) g(a)+\operatorname{tg}(b)) \\
& \quad+((1-t) f(a)+t f(b)) g(a)] \\
& +\frac{1-t}{6}[((1-t) f(a)+t f(b)) g(b) \\
& \quad+f(b)((1-t) g(a)+\operatorname{tg}(b))] \\
& =\frac{t}{6}[2(1-t) f(a) g(a)+t N(a, b)] \\
& \quad+\frac{1-t}{6}[2 t f(b) g(b)+(1-t) N(a, b)] \\
& =\frac{t(1-t)}{3} M(a, b)+\frac{(1-t)^{2}+t^{2}}{6} N(a, b),
\end{aligned}
$$

we have

$$
\begin{aligned}
L(t) \leq & \frac{1-t+t^{2}}{3} M(a, b)+\frac{(1-t) t}{3} N(a, b) \\
& +\frac{t(1-t)}{3} M(a, b)+\frac{(1-t)^{2}+t^{2}}{6} N(a, b) \\
= & \frac{1}{3} M(a, b)+\frac{1}{6} N(a, b)
\end{aligned}
$$

which completes the proof.

Remark 4. Applying Theorem 3 for $L(0)=L(1)=(1 /$ 3) $M(a, b)+(1 / 6) N(a, b)$, we get the result in Theorem 1 . So, we conclude that our results give an improvement of Theorem 1.

Corollary 5. With notations above, one has the following inequality:

$$
\begin{aligned}
\frac{1}{b-a} \int_{a}^{b} f(x) g(x) d x & \leq \inf _{0 \leq t \leq 1} L(t) \leq \sup _{0 \leq t \leq 1} L(t) \\
& \leq \frac{1}{3} M(a, b)+\frac{1}{6} N(a, b)
\end{aligned}
$$

where $L(t)$ is defined in Theorem 3.

Theorem 6. Let $f$ and $g$ be real-valued, nonnegative, and convex functions on $[a, b]$. Then,

$$
\begin{aligned}
2 f\left(\frac{a+b}{2}\right) g\left(\frac{a+b}{2}\right) \\
\leq \frac{1}{b-a} \int_{a}^{b} f(x) g(x) d x \\
+\frac{1}{12}\left[N\left(a, \frac{a+b}{2}\right)+N\left(\frac{a+b}{2}, a\right)\right] \\
+\frac{1}{6}\left[N\left(\frac{a+b}{2}, \frac{a+b}{2}\right)+N(a, b)\right]
\end{aligned}
$$

$$
\begin{aligned}
\leq & \frac{1}{b-a} \int_{a}^{b} f(x) g(x) d x \\
& +\frac{1}{6} M(a, b)+\frac{1}{3} N(a, b),
\end{aligned}
$$

where $M(a, b)=f(a) g(a)+f(b) g(b)$ and $N(a, b)=$ $f(a) g(b)+f(a) g(b)$.

Proof. Since $f$ and $g$ are convex on $[a, b]$ and nonnegative, for $t \in[0,1]$, we observe that

$$
\begin{aligned}
& 2 f\left(\frac{a+b}{2}\right) g\left(\frac{a+b}{2}\right) \\
& =2 f\left(\frac{t((a+b) / 2)+(1-t) b}{2}\right. \\
& \left.+\frac{(1-t) a+t((a+b) / 2)}{2}\right) \\
& \times g\left(\frac{t((a+b) / 2)+(1-t) b}{2}\right. \\
& \left.+\frac{(1-t) a+t((a+b) / 2)}{2}\right) \\
& \leq \frac{1}{2}\left[f\left(t \frac{a+b}{2}+(1-t) b\right)+f\left((1-t) a+t \frac{a+b}{2}\right)\right] \\
& \times\left[g\left(t \frac{a+b}{2}+(1-t) b\right)+g\left((1-t) a+t \frac{a+b}{2}\right)\right] \\
& \leq \frac{1}{2}\left[f\left(t \frac{a+b}{2}+(1-t) b\right) g\left(t \frac{a+b}{2}+(1-t) b\right)\right. \\
& \left.+f\left((1-t) a+t \frac{a+b}{2}\right) g\left((1-t) a+t \frac{a+b}{2}\right)\right] \\
& +\frac{1}{2}\left[\left(t f\left(\frac{a+b}{2}\right)+(1-t) f(b)\right)\right. \\
& \times\left((1-t) g(a)+\operatorname{tg}\left(\frac{a+b}{2}\right)\right) \\
& +\left((1-t) f(a)+t f\left(\frac{a+b}{2}\right)\right) \\
& \left.\times\left(\operatorname{tg}\left(\frac{a+b}{2}\right)+(1-t) g(b)\right)\right] \\
& =\frac{1}{2}\left[f\left(t \frac{a+b}{2}+(1-t) b\right) g\left(t \frac{a+b}{2}+(1-t) b\right)\right. \\
& \left.+f\left((1-t) a+t \frac{a+b}{2}\right) g\left((1-t) a+t \frac{a+b}{2}\right)\right] \\
& +\frac{1}{2}\left[t(1-t) f\left(\frac{a+b}{2}\right) g(a)\right. \\
& +t^{2} f\left(\frac{a+b}{2}\right) g\left(\frac{a+b}{2}\right) \\
& +(1-t)^{2} f(b) g(a)+(1-t) t f(b) g\left(\frac{a+b}{2}\right)
\end{aligned}
$$




$$
\begin{aligned}
& +(1-t)^{2} f(a) g(b)+(1-t) t f(a) g\left(\frac{a+b}{2}\right) \\
& +t(1-t) f\left(\frac{a+b}{2}\right) g(b) \\
& \left.+t^{2} f\left(\frac{a+b}{2}\right) g\left(\frac{a+b}{2}\right)\right] \\
& =\frac{1}{2}\left[f\left(t \frac{a+b}{2}+(1-t) b\right) g\left(t \frac{a+b}{2}+(1-t) b\right)\right. \\
& \left.+f\left((1-t) a+t \frac{a+b}{2}\right) g\left((1-t) a+t \frac{a+b}{2}\right)\right] \\
& +\frac{1}{2}\left[t(1-t) N\left(a, \frac{a+b}{2}\right)+t^{2} N\left(\frac{a+b}{2}, \frac{a+b}{2}\right)\right. \\
& \left.+(1-t)^{2} N(a, b)+t(1-t) N\left(\frac{a+b}{2}, b\right)\right] .
\end{aligned}
$$

Integrating both sides of the previous inequality with respect to $t$ over $[0,1]$, we obtain

$$
\begin{aligned}
& 2 f\left(\frac{a+b}{2}\right) g\left(\frac{a+b}{2}\right) \\
& \leq \frac{1}{2}\left[\int_{0}^{1} f\left(t \frac{a+b}{2}+(1-t) b\right)\right. \\
& \times g\left(t \frac{a+b}{2}+(1-t) b\right) d t \\
& +\int_{0}^{1} f\left((1-t) a+t \frac{a+b}{2}\right) \\
& \left.\times g\left((1-t) a+t \frac{a+b}{2}\right) d t\right] \\
& +\frac{1}{2}\left[\frac{1}{6} N\left(a, \frac{a+b}{2}\right)+\frac{1}{3} N\left(\frac{a+b}{2}, \frac{a+b}{2}\right)\right. \\
& \left.+\frac{1}{3} N(a, b)+\frac{1}{6} N\left(\frac{a+b}{2}, b\right)\right] \\
& =\frac{1}{2}\left[\frac{2}{b-a} \int_{(a+b) / 2}^{b} f(x) g(x) d x\right. \\
& \left.+\frac{2}{b-a} \int_{a}^{(a+b) / 2} f(x) g(x) d x\right] \\
& +\frac{1}{2}\left[\frac{1}{6} N\left(a, \frac{a+b}{2}\right)+\frac{1}{3} N\left(\frac{a+b}{2}, \frac{a+b}{2}\right)\right. \\
& \left.+\frac{1}{3} N(a, b)+\frac{1}{6} N\left(\frac{a+b}{2}, b\right)\right] \\
& =\frac{1}{b-a} \int_{a}^{b} f(x) g(x) d x
\end{aligned}
$$

$$
\begin{aligned}
& +\frac{1}{12} N\left(a, \frac{a+b}{2}\right)+\frac{1}{6} N\left(\frac{a+b}{2}, \frac{a+b}{2}\right) \\
& +\frac{1}{6} N(a, b)+\frac{1}{12} N\left(\frac{a+b}{2}, b\right) .
\end{aligned}
$$

On the other hand, we have

$$
\begin{aligned}
N(a, & \left.\frac{a+b}{2}\right) \\
& =f(a) g\left(\frac{a+b}{2}\right)+f\left(\frac{a+b}{2}\right) g(a) \\
& \leq \frac{1}{2}[f(a)(g(a)+g(b))+(f(a)+f(b)) g(a)] \\
& =f(a) g(a)+\frac{1}{2} N(a, b) .
\end{aligned}
$$

By a similar way, we obtain

$$
\begin{aligned}
& N\left(\frac{a+b}{2}, b\right) \\
& \quad=f(b) g\left(\frac{a+b}{2}\right)+f\left(\frac{a+b}{2}\right) g(b) \\
& \quad \leq \frac{1}{2}[f(b)(g(a)+g(b))+(f(a)+f(b)) g(b)] \\
& \quad=f(b) g(b)+\frac{1}{2} N(a, b), \\
& N\left(\frac{a+b}{2}, \frac{a+b}{2}\right)=2 f\left(\frac{a+b}{2}\right) g\left(\frac{a+b}{2}\right) \\
& \quad \leq \frac{1}{2}[(g(a)+g(b))(f(a)+f(b))] \\
& \quad=\frac{1}{2} M(a, b)+\frac{1}{2} N(a, b) .
\end{aligned}
$$

So,

$$
\begin{aligned}
\frac{1}{12} N\left(a, \frac{a+b}{2}\right)+\frac{1}{6} N\left(\frac{a+b}{2}, \frac{a+b}{2}\right) \\
+\frac{1}{6} N(a, b)+\frac{1}{12} N\left(\frac{a+b}{2}, b\right) \\
\leq \frac{1}{12}\left[f(a) g(a)+\frac{1}{2} N(a, b)\right. \\
\left.\quad+f(b) g(b)+\frac{1}{2} N(a, b)\right] \\
+\frac{1}{6}\left[\frac{1}{2} M(a, b)+\frac{1}{2} N(a, b)\right]+\frac{1}{6} N(a, b) \\
=\frac{1}{6} M(a, b)+\frac{1}{3} N(a, b),
\end{aligned}
$$

which yields the desired result. 


\section{Conclusion}

In this paper, we establish some new Hermite-Hadamard type inequalities for products of convex functions. The results obtained in this work are the refinements of the present results. An interesting topic is whether we can use the methods in this paper to refine the Hermite-Hadamard inequality for coordinated convex functions.

\section{Acknowledgment}

This work is supported by the Youth Project of Chongqing Three Gorges University of China.

\section{References}

[1] S. Abramovich, G. Farid, and J. Pečarić, "More about HermiteHadamard inequalities, Cauchy's means, and superquadracity," Journal of Inequalities and Applications, vol. 2010, Article ID 102467, 14 pages, 2010.

[2] A. Barani, S. Barani, and S. S. Dragomir, "Refinements of Hermite-Hadamard inequalities for functions when a power of the absolute value of the second derivative is P-convex," Journal of Applied Mathematics, vol. 2012, Article ID 615737, 10 pages, 2012.

[3] M. Bessenyei and Z. Páles, "Hadamard-type inequalities for generalized convex functions," Mathematical Inequalities \& Applications, vol. 6, no. 3, pp. 379-392, 2003.

[4] S. S. Dragomir, "Hermite-Hadamard's type inequalities for operator convex functions," Applied Mathematics and Computation, vol. 218, no. 3, pp. 766-772, 2011.

[5] S. S. Dragomir, "Hermite-Hadamard's type inequalities for convex functions of selfadjoint operators in Hilbert spaces," Linear Algebra and Its Applications, vol. 436, no. 5, pp. 1503-1515, 2012.

[6] A. El Farissi, "Simple proof and refinement of HermiteHadamard inequality," Journal of Mathematical Inequalities, vol. 4, no. 3, pp. 365-369, 2010.

[7] X. Gao, "A note on the Hermite-Hadamard inequality," Journal of Mathematical Inequalities, vol. 4, no. 4, pp. 587-591, 2010.

[8] B. G. Pachpatte, "On some inequalities for convex functions," RGMIA Research Report Collection, vol. 6, 2003.

[9] B. G. Pachpatte, "A note on integral inequalities involving two log-convex functions," Mathematical Inequalities \& Applications, vol. 7, no. 4, pp. 511-515, 2004.

[10] U. S. Kirmaci, M. Klaričić Bakula, M. E. Özdemir, and J. Pečarić, "Hadamard-type inequalities for s-convex functions," Applied Mathematics and Computation, vol. 193, no. 1, pp. 26-35, 2007.

[11] M. Z. Sarikaya, A. Saglam, and H. Yildirim, "On some Hadamard-type inequalities for $h$-convex functions," Journal of Mathematical Inequalities, vol. 2, no. 3, pp. 335-341, 2008.

[12] V. Bacak and R. Türkmen, "New inequalities for operator convex functions," Journal of Inequalities and Applications, vol. 2013, article 190, 2013.

[13] E. Set, M. E. Özdemir, and S. S. Dragomir, "On the HermiteHadamard inequality and other integral inequalities involving two functions," Journal of Inequalities and Applications, vol. 2010, Article ID 148102, 9 pages, 2010. 


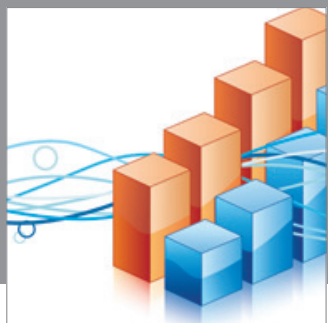

Advances in

Operations Research

mansans

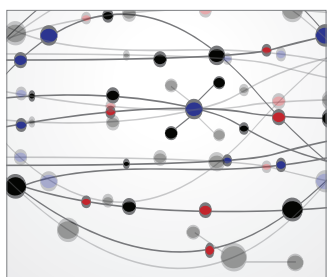

The Scientific World Journal
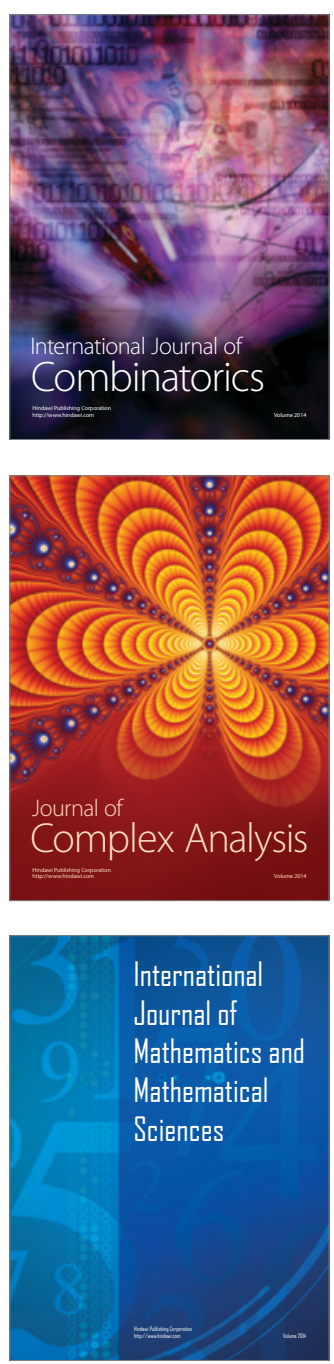
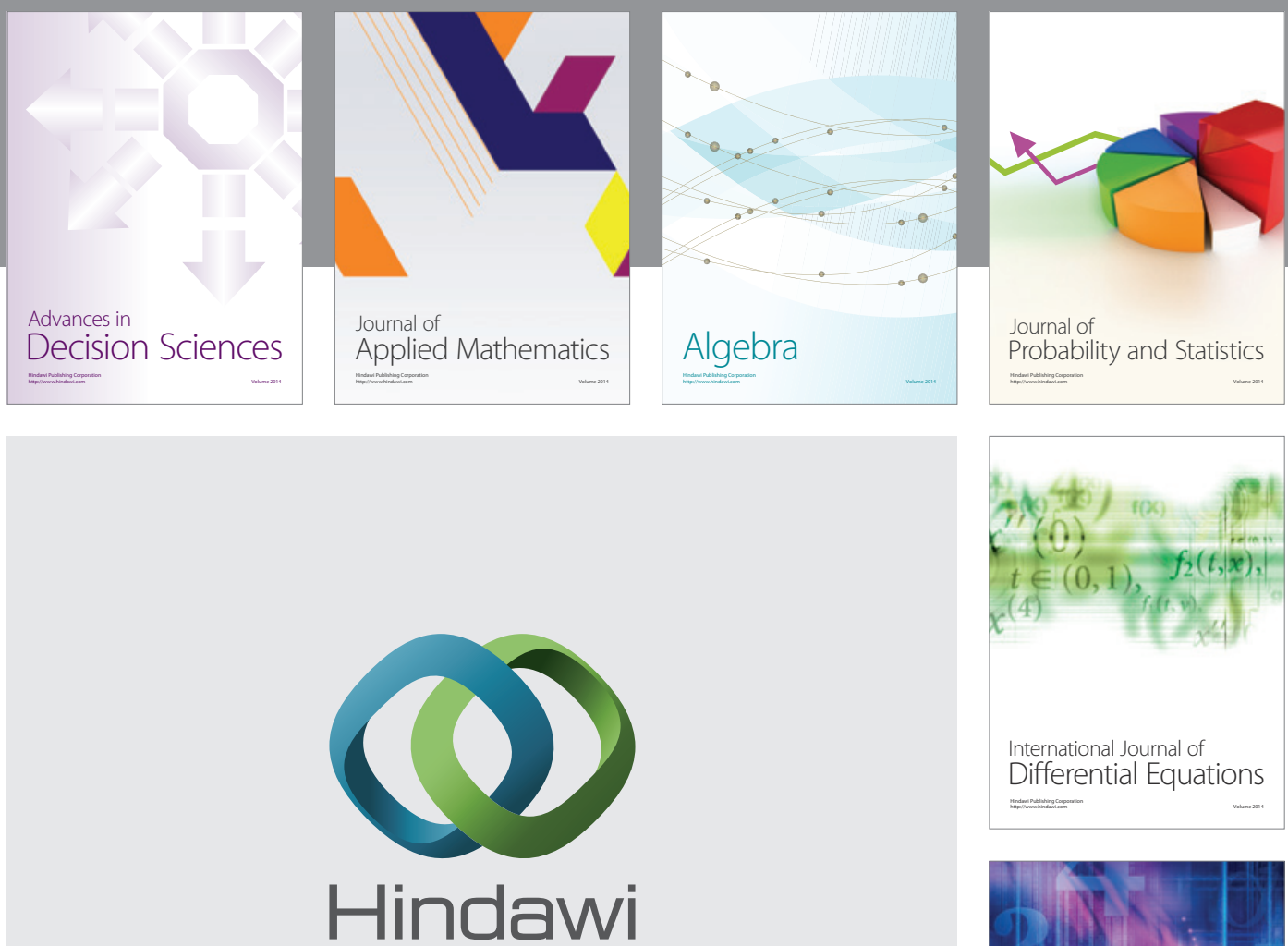

Submit your manuscripts at http://www.hindawi.com
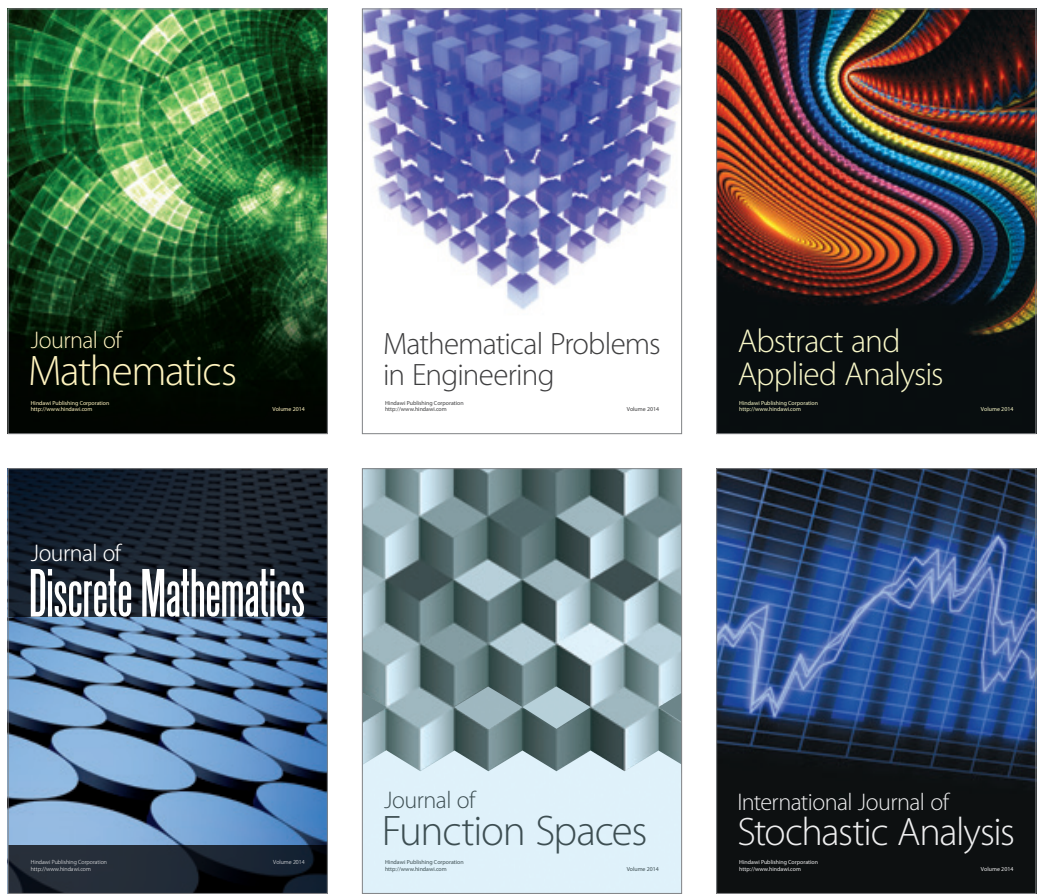

Journal of

Function Spaces

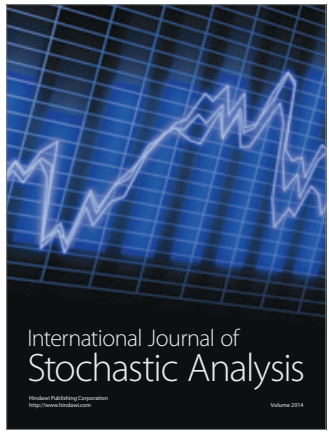

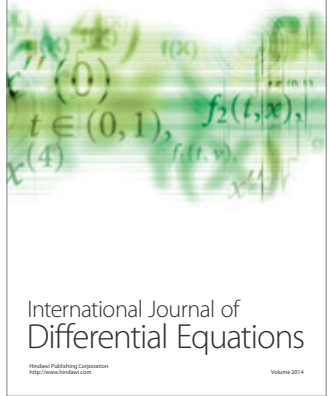
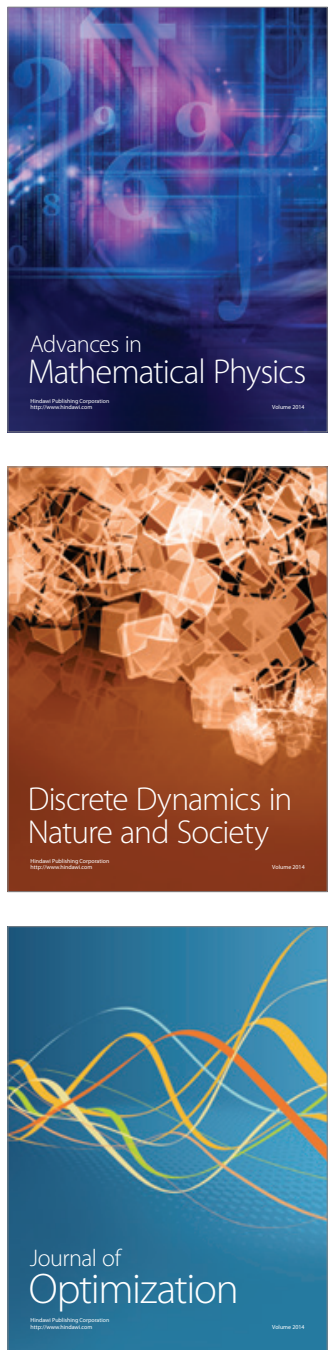